\title{
Efeito da prescrição de caminhada sem su- pervisão da prática num parque público de São Paulo
}

\author{
Effects of walking prescription executed \\ without supervision in a public park \\ of São Paulo
}

Rev Bras Ativ Fis Saúde p. 423-433

DOI: http://dx.doi.org/10.12820/2317-

1634.2012v17n5p423

1 Escola de Educação Física e Esporte, Universidade de São Paulo, São Paulo, SP Brasil

2 LAHAM - Laboratório de Hemodinâmica da Atividade Motora

\author{
Paulo Panisi ${ }^{1,2}$ \\ Paula Roberta Pádua ${ }^{1,2}$ \\ Victor Matheus Silva Martins ${ }^{1,2}$ \\ Julia Albino ${ }^{1,2}$ \\ Leandro Campos de Brito ${ }^{1,2}$ \\ Teresa Bartholomeu ${ }^{1,2}$ \\ Taís Tinucci ${ }^{1,2}$ \\ Cláudia Lúcia de Moraes Forjaz ${ }^{1,2}$
}

\section{Resumo}

Este estudo objetivou avaliar o efeito da prescrição de caminhada, realizada sem supervisão da execução, na aptidão física e no risco cardiovascular de usuários de um parque público. Para tanto, 113 voluntários foram avaliados por um questionário de risco cardiovascular; medidas antropométricas, metabólicas e cardiovasculares; e testes de aptidão física. Em seguida, receberam uma prescrição individualizada de caminhada, que realizaram sem supervisão direta de um profissional e foram reavaliados entre 3 e 9 meses. Após a intervenção, 88 pessoas relataram ter seguido a prescrição. Nelas, houve redução do índice de massa corporal $\left(-0,3 \pm 1,0 \mathrm{~kg} / \mathrm{m}^{2}\right.$, $\mathrm{P} \leq 0,05)$ e da pressão arterial diastólica $(-2,4 \pm 8,1 \mathrm{mmHg}, \mathrm{P}<0,05)$. Houve também aumento da aptidão aeróbica, potência abdominal e das flexibilidades de ombro e lombar $(+10,3 \pm 17,8$ passadas, $+1,3 \pm 4,8$ abdominais, $+1,16 \pm 2,45 \mathrm{~cm},+1,15 \pm 4,60 \mathrm{~cm}$, respectivamente, $\mathrm{P}<0,05)$. No grupo que não seguiu as recomendações, não houve benefícios. Desta forma, foi possível concluir que a prescrição de caminhada sem supervisão da execução foi efetiva em melhorar o risco cardiovascular e a aptidão física dos usuários que seguiram as orientações.

\section{Palavras-chave}

Prescrição; Exercício; Fatores de risco; Aptidão física; Local público.

\begin{abstract}
This study aimed to evaluate the effect of walking prescription, executed without direct supervision, on physical fitness and cardiovascular risk of the users of a public park. One bundred and thirteen volunteers were evaluated by a cardiovascular risk questionnaire; anthropometric, metabolic and cardiovascular measurements, and fitness tests. Afterwards, they received an individualized prescription of walking to execute without supervision, and were reevaluated between 3 and 9 months. After the intervention, 88 subjects reported to have followed the orientations. In them, body mass index $(-0.3$ $\left.\pm 1.0 \mathrm{~kg} / \mathrm{m}^{2}, P<0.05\right)$ and diastolic blood pressure decreased $(-2.4 \pm 8.1 \mathrm{mmHg}, P<0.05)$. In addition, aerobic fitness, abdominal power, and shoulder and lumbar flexibilities increased $(+10.3 \pm 17.8$ steps, $+1.3 \pm 4.8$ repetitions, $+1.16 \pm 2.45 \mathrm{~cm},+1.15 \pm 4.60 \mathrm{~cm}$, respectively, $P<0.05)$. In the subjects who did not follow the recommendations, no benefit was observed. Thus, it is possible to conclude that, in a public park, the prescription of unsupervised walking was effective for improving cardiovascular risk and physical fitness in subjects who followed the orientations.
\end{abstract}

\section{Keywords}

Prescription; Exercise; Risk factors; Fitness physical; Public place. 


\section{INTRODUÇÃO}

As doenças cardiovasculares são a principal causa de morte da população adulta brasileira, representando cerca de $30 \%$ das mortes no país e atingindo números maiores em São Paulo ${ }^{1}$. Dentre os Fatores de Risco Cardiovascular (FRC) que predispõem ao desenvolvimento destas doenças, a inatividade física é um dos principais, apresentando prevalências que variam de 11 a $18 \%$ nos brasileiros $\geq 18$ anos $^{2}$ quando medidas por inquérito telefônico, mas podendo ser bem maiores se avaliadas por métodos mais diretos, o que demonstra a importância de seu combate.

A prática regular de exercício físico está associada à redução da morbidade e da mortalidade cardiovascular ${ }^{3}$, pois esta prática é capaz de: a) reduzir a pressão arterial sistólica (PAS) e diastólica (PAD) em, $-3,3$ e -3,5mmHg ${ }^{4}$ respectivamente; b) melhorar a sensibilidade à insulina, mantendo o controle glicêmico, diminuindo a hemoglobina glicosilada e prevenindo o diabetes mellitus 5 ; c) modificar o perfil lipídico, reduzindo as concentrações de triglicérides, LDL - colesterol e colesterol total na ordem de $-4 \%,-5 \%$ e $-1 \%$, respectivamente, e aumentando a de HDL colesterol em $+5 \%{ }^{6}$; e d) auxiliar no controle da obesidade, reduzindo a quantidade de gordura, principalmente na região abdominal, o que resulta na redução da massa corporal, do índice de massa corporal (IMC), da circunferência da cintura (CC) e do índice cintura-quadril (ICQ) ${ }^{5}$.

Devido a estes benefícios, campanhas de incentivo à prática regular de atividades físicas (AF) têm sido criadas e têm alcançado suas metas ${ }^{7,8}$. Porém, os espaços públicos procurados para esta prática não costumam dispor de recursos humanos para avaliar e orientar os usuários quanto ao EF adequado ${ }^{9}$, e a prática inadequada pode não trazer os benefícios esperados ${ }^{10}$. Além disso, exercícios intensos aumentam o risco da ocorrência de eventos cardiovasculares durante sua execução ${ }^{10}$. Para minimizar este risco, o American College of Sports Medicine (ACSM) elaborou um modelo de triagem cardiovascular para a prática de $\mathrm{EF}^{11}$, que é recomendado até hoje $\mathrm{e}^{10}$. Aplicando este modelo em um parque público, observamos uma alta prevalência de pessoas com risco moderado a alto se exercitando ${ }^{12}$. Além disso, demonstramos que grande parte dos praticantes de $\mathrm{EF}$ nos parques públicos não controla a intensidade do exercício de forma efetiva ${ }^{13}$. Estes resultados realçam a importância de uma triagem prévia e da prescrição adequada de EF para os praticantes de locais públicos.

Para minimizar este quadro, alguns programas têm sido criados para orientar/ prescrever o EF nestes locais, como o projeto Exercício e Coração ${ }^{14}$. Por terem uma característica populacional, estes programas prescrevem o EF, mas não supervisionam sua prática, o que possibilita o atendimento a um grande número de pessoas com baixo custo financeiro ${ }^{15}$. Entretanto, embora a eficácia do EF supervisionado já esteja comprovada, a efetividade de programas não supervisionados foi menos investigada, e os estudos existentes ${ }^{15,16}$ envolveram situações específicas de pesquisa e não situações reais de atuação prática junto à população.

Dentre os diversos EF que podem ser utilizadas em programas de intervenção populacional, a caminhada merece destaque ${ }^{17}$, pois melhora a aptidão aeróbica e promove benefícios à saúde (redução da pressão arterial, auxílio no controle de peso, entre outros) em pessoas previamente sedentárias. Além disso, ela apresenta facilidade de execução (não precisa de local nem equipamentos especiais), baixo risco e taxa de adesão maior que outras AF. Porém, seu efeito quando aplicada numa situação real de prática sem supervisão ainda não foi demonstrado. 
Assim, este estudo foi desenvolvido num programa de caráter populacional, o Projeto Exercício e Coração, com o objetivo de verificar o efeito da orientação/ prescrição de caminhada, realizada sem supervisão da prática sobre a aptidão física e os FRC de usuários de um parque público.

\section{MÉTODOS}

\section{Amostra}

Esta pesquisa foi desenvolvida dentro dos padrões éticos exigidos pela declaração de Helsinque de 1964 e de acordo com a resolução 196/96 do Ministério da Saúde. O projeto foi aprovado pela Comissão de Ética da Instituição que conduziu o estudo (protocolo 2002/10).

A coleta de dados ocorreu no Parque Fernando Costa em São Paulo de Abril de 2002 a Maio de 2008. O projeto foi divulgado para os indivíduos adultos que frequentavam o parque através de contato direto, entrega de folhetos, chamadas no jornal do parque e por eventos realizados em fins de semana. Todos os indivíduos que tiveram interesse em participar agendaram uma avaliação. Eles foram esclarecidos sobre a pesquisa e assinaram o termo de consentimento aprovado pelo Comitê de Ética.

Os indivíduos foram inicialmente avaliados, receberam uma prescrição e foram convidados para uma reavaliação. No período do estudo, foram realizadas 616 avaliações e 183 reavaliações, sendo que alguns indivíduos fizeram mais de uma reavaliação. Assim, para este estudo foi considerada apenas a primeira reavaliação de cada indivíduo, desde que ela tivesse ocorrido num intervalo de 3 a 9 meses da avaliação. Dessa forma, foram analisados os dados de 121 reavaliações que seguiram este critério.

As avaliações e reavaliações foram previamente agendadas e duraram aproximadamente $90 \mathrm{~min}$. Elas foram realizadas pela manhã com os indivíduos em jejum de $12 \mathrm{~h}$ e sem fazerem EF nas $24 \mathrm{~h}$ anteriores. As avaliações foram realizadas por monitores, alunos de graduação em educação física, devidamente treinados pelos coordenadores responsáveis.

A avaliação foi composta, inicialmente, por um questionário sobre o risco cardiovascular, incluindo: dados pessoais; presença de sintomas, doenças ou FRC conhecidos; e uso regular de medicamentos que afetassem o sistema cardiovascular. Os níveis de $\mathrm{AF}$ de lazer foram questionados (se o indivíduo fazia alguma $\mathrm{AF}$ de lazer? E em caso positivo: qual, quantas vezes por semana, por quanto tempo e com que intensidade?). Foram considerados ativos, os indivíduos que somavam mais de $150 \mathrm{~min} / \mathrm{semana}$ de $\mathrm{AF}$ de lazer e não ativos aqueles que realizavam menos que isso. Em seguida, foram realizadas medidas dos FRC, sendo avaliados: a) índices antropométricos: a massa corporal foi medida numa balança portátil (Welmy), a estatura num estadiômetro e a circunferência da cintura e do quadril por uma fita métrica, sendo calculados o IMC e ICQ; b) índices metabólicos: a glicemia e a colesterolemia foram medidas pela punção digital e monitores automáticos (Glucometer 4 - Accu-Check e Accurtrend GC - Roche, respectivamente); e c) índices hemodinâmicos: a PAS e a PAD foram medidas em triplicada pelo método auscultatório após repouso sentado de $5 \mathrm{~min}$ (quando necessário, houve a correção dos valores pela circunferência do braço) e a $\mathrm{FC}$ de repouso foi medida pela palpação da artéria radial por $15 \mathrm{~s}$. Para finalizar, foram executados testes de aptidão física, que incluíram; a) a aptidão cardiorrespiratória avaliada pelo teste de 
marcha estacionária ${ }^{18}$; b) a potência de membros superiores pelo teste de flexão e extensão de $\operatorname{cotovelo~}^{18}$; c) a força de membros inferiores pelo teste de impulsão vertical $^{19}$; d) a potência abdominal pelo teste da American Heart Association ${ }^{20}$; e) a flexibilidade lombar pelo teste de sentar e alcançar de Wells ${ }^{21}$; e f) a flexibilidade de ombros por meio do teste de Rikli \& Jones ${ }^{18}$.

Ao término da avaliação foi realizada a orientação/prescrição da caminhada. Porém, os cardiopatas e os indivíduos que apresentavam sintomas foram inicialmente encaminhados ao médico e a prescrição só foi realizada após a permissão deste profissiona ${ }^{10}$. Os indivíduos foram orientados a caminhar, no mínimo, 3 vezes/semana por 30 min em intensidade moderada (50 a 70\% da FC de reserva para os sedentários, cardiopatas, hipertensos e obesos, e 60 a $80 \%$ para os indivíduos saudáveis e já condicionados ${ }^{22}$. Para os indivíduos que possuíam um teste ergométrico máximo recente (menos de 6 meses), a FC máxima utilizada na fórmula foi a atingida no teste, nos demais sujeitos, utilizou-se a fórmula 220-idade. Para os indivíduos que faziam uso de betabloqueadores e não tinham teste ergométrico com esta droga e para aqueles que não conseguiam medir a FC durante a caminhada foi indicado o controle da intensidade pela respiração, ou seja, caminhar o mais rápido possível desde que conseguissem conversar durante o esforço ${ }^{22}$. Os indivíduos foram instruídos a medir a $\mathrm{FC}$ no meio e no final da caminhada.

Os indivíduos participaram de 3 sessões de EF supervisionado realizadas, conforme a disponibilidade do participante, num prazo máximo de 2 semanas. Nestas sessões, os voluntários aprenderam a medir a FC pela palpação da artéria radial e foram instruídos a manter a intensidade da caminhada na faixa prescrita ${ }^{22}$. Para finalizar, os sujeitos receberam um folheto com exercícios de alongamento e foram orientados a realizá-los no início e ao final das sessões de exercício. Estes alongamentos também poderiam ser realizados em aulas oferecidas 3 vezes por semana. Para finalizar, os voluntários receberam informações gerais sobre vestimenta, alimentação e hidratação adequadas para o exercício.

Todos os indivíduos foram convidados a retornar para a reavaliação após 3 meses. Nesta reavaliação, eles foram questionados quanto às modificações que haviam ocorrido entre a avaliação e a reavaliação: se haviam seguido a orientação de caminhada (volume, frequência e intensidade), se haviam mudado as outras AF que faziam, se haviam retornado ao médico e se houve alguma mudança em seu quadro clínico. Em seguida, todas as medidas e testes realizados na avaliação foram repetidos e uma prescrição atualizada de caminhada (novo volume ou nova intensidade, quando adequado) foi fornecida.

Com os dados da reavaliação, os indivíduos foram divididos em 2 grupos. $\mathrm{O}$ primeiro foi formado por quem seguiu (S) totalmente (frequência, duração e intensidade) ou pelo menos parcialmente (seguiu pelo menos um dos parâmetros) a prescrição. $\mathrm{O}$ segundo foi formado pelos indivíduos que relataram não terem seguido (NS) as recomendações.

Para a análise estatística, a distribuição normal dos dados foi checada pelos testes de Shapiro-Wilk e Komolgorov. As comparações foram feitas pelo teste T-Student para amostras repetidas ou não repetidas ou pelos testes de Wilcoxon e Mann-Whitney, conforme o caso. Todas as análises foram realizadas através do pacote estatístico SPSS 13.0. O nível de significância aceito foi de $\mathrm{P} \leq 0,05$ e os dados estão apresentados como média \pm desvio padrão. 


\section{RESULTADOS}

A amostra inicial foi formada por 121 indivíduos, dos quais 8 não conseguiram responder de forma precisa se haviam ou não seguido as recomendações. Assim, a amostra final foi de 113 sujeitos. Destes, 68 (56\%) disseram ter seguido integralmente as recomendações e 20 (17\%) apenas parcialmente, de modo que o grupo S foi formado por 88 (73\%) voluntários. Os 25 (21\%) sujeitos que disseram não ter seguido as recomendações formaram o grupo NS. Nos que seguiram parcialmente, os principais motivos foram falta de tempo (20\%) e preguiça (10\%). Nos que não seguiram, o principal motivo foi falta de tempo (32\%). Considerando-se os que seguiram integralmente, $43 \%$ disseram ter acompanhado a intensidade de treinamento pela medida da $\mathrm{FC}$ e aqueles que não mediram a $\mathrm{FC}$ relataram não conseguir executar este procedimento de medição (33\%). O tempo médio de seguimento dos grupos foi $181 \pm 49$ dias no S e $194 \pm 48$ dias no NS.

Os grupos S e NS apresentavam características semelhantes (Tabela 1).

Tabela 1 - Características do grupo que seguiu (S) e que não seguiu (NS) a prescrição.

\begin{tabular}{llcc}
\hline Característica & Feminino & $\begin{array}{c}\mathrm{S} \\
\mathrm{n}(\%)\end{array}$ & $\begin{array}{c}\mathrm{N}(\%) \\
\mathrm{n}(\%)\end{array}$ \\
\hline Sexo & Masculino & $58(66 \%)$ & $17(68 \%)$ \\
\hline Idade & $30-39$ & $30(34 \%)$ & $8(32 \%)$ \\
\hline & $40-49$ & $3(3 \%)$ & $2(8 \%)$ \\
\hline & $50-59$ & $9(10 \%)$ & $1(4 \%)$ \\
\hline Risco Cardiovascular & $60-69$ & $25(28 \%)$ & $7(28 \%)$ \\
\hline & Mais de 70 & $29(33 \%)$ & $10(40 \%)$ \\
\hline & Doença Cardíaca & $22(28 \%)$ & $5(20 \%)$ \\
\hline & Hereditariedade & $11(13 \%)$ & $5(20 \%)$ \\
\hline & Hipertensão & $30(34 \%)$ & $8(32 \%)$ \\
\hline & Diabetes & $26(30 \%)$ & $10(40 \%)$ \\
\hline & Hipercolesterolemia & $27(31 \%)$ & $9(36 \%)$ \\
\hline & Tabagismo & $5(6 \%)$ & $1(4 \%)$ \\
\hline
\end{tabular}

Comparação pelo teste de Qui-quadrado. Todas P>0.05

$\mathrm{Na}$ avaliação, os grupos apresentaram medidas de risco cardiovascular e de aptidão física semelhantes, exceto pelo IMC que foi maior e a flexibilidade de ombros que foi menor no grupo NS que no S (Tabela 2).

$\mathrm{Na}$ reavaliação, a massa corporal, o IMC e a PAD diminuíram no grupo $\mathrm{S}$, enquanto que no grupo NS, o IMC e a glicemia aumentaram. Em relação à aptidão física, no grupo $\mathrm{S}$, todas as capacidades físicas (exceto a potência de membros superiores e a força de membros inferiores) aumentaram na reavaliação, enquanto que no grupo NS, não houve nenhuma modificação significante.

Para verificar se os efeitos observados no grupo $\mathrm{S}$ diferiam segundo o gênero, a idade e o nível inicial de AF, os sujeitos deste grupo foram separados em: a) homens $(\mathrm{n}=30)$ e mulheres $(\mathrm{n}=58) ; \mathrm{b})$ meia idade (MI $-<60$ anos, $\mathrm{n}=37)$ e idosos (ID $-\geq 60$ anos, $\mathrm{n}=51$ ) e c) ativos ( $\mathrm{A}-\geq 150 \mathrm{~min} / \mathrm{semana}, \mathrm{n}=53$ ) e não ativos (NA, <150min/ sem, $\mathrm{n}=35$ ). As respostas observadas nestes subgrupos estão apresentadas na Tabela 3 .

$\mathrm{Na}$ avaliação, os homens tinham maior massa, CC, ICQ, PAS e PAD que as mulheres, mas menor FC. Eles apresentaram maior potência abdominal e força 
Tabela 2 - Valores medidos na avaliação e mudanças obtidas na reavaliação no grupo que seguiu (S) e que não seguiu (NS) a prescrição de caminhada.

\begin{tabular}{lcccc}
\hline & \multicolumn{2}{c}{ Avaliação } & \multicolumn{2}{c}{ Diferença na Reavaliação } \\
\hline & $\begin{array}{c}\mathrm{S} \\
(\mathrm{n}=88)\end{array}$ & $\begin{array}{c}\mathrm{NS} \\
(\mathrm{n}=25)\end{array}$ & $\begin{array}{c}\mathrm{S} \\
(\mathrm{n}=88)\end{array}$ & $\begin{array}{c}\text { NS } \\
(\mathrm{n}=25)\end{array}$ \\
\hline Massa (kg) & $68,5 \pm 11,8$ & $72,6 \pm 14,2$ & $-0,7 \pm 2,7 *$ & $0,1 \pm 2,2$ \\
\hline IMC (kg/m2) & $26,5 \pm 3,3$ & $28,6 \pm 5,2+$ & $-0,3 \pm 1,0 *$ & $0,1 \pm 0,9 *$ \\
\hline Cintura (cm) & $93 \pm 10$ & $97 \pm 14$ & $-0,6 \pm 5,3$ & $0,5 \pm 4,0$ \\
\hline Quadril (cm) & $102 \pm 8$ & $104 \pm 9$ & $-0,7 \pm 3,2$ & $0,3 \pm 2,8$ \\
\hline ICQ & $0,91 \pm 0,07$ & $0,93 \pm 0,10$ & $0,00 \pm 0,06$ & $0,00 \pm 0,06$ \\
\hline Glicose (mg /dL) & $100 \pm 18$ & $94 \pm 13$ & $0,3 \pm 12,1$ & $7,5 \pm 12,0 *$ \\
\hline Colesterol (mg/dL) & $198 \pm 36$ & $211 \pm 36$ & $-2,2 \pm 33,3$ & $-2,8 \pm 30,7$ \\
\hline PA Sistólica (mmHg) & $128 \pm 19$ & $128 \pm 20$ & $-2,7 \pm 13,3$ & $-5,6 \pm 18,9$ \\
\hline PA Diastólica (mmHg) & $81 \pm 9$ & $82 \pm 11$ & $-2,4 \pm 8,1 *$ & $-3,5 \pm 9,0$ \\
\hline FC (bat/min) & $65 \pm 10$ & $65 \pm 8$ & $0,7 \pm 8,2$ & $1,1 \pm 6,8$ \\
\hline Aptidão Cardio. (passadas) & $103 \pm 22$ & $103 \pm 24$ & $10,3 \pm 17,8 *$ & $3,6 \pm 17,6$ \\
\hline Potência MMSS (repetiç̃̃es) & $20 \pm 4$ & $19 \pm 5$ & $0,1 \pm 4,3$ & $1,0 \pm 4,4$ \\
\hline Potência ABD (abdominais) & $19 \pm 5$ & $18 \pm 5$ & $1,3 \pm 4,8 *$ & $0,9 \pm 4,0$ \\
\hline Força MMII (cm) & $19 \pm 7$ & $19 \pm 5$ & $0,5 \pm 3,4$ & $1,0 \pm 3,0$ \\
\hline Flexibilidade Ombros (cm) & $-0,5 \pm 7,4$ & $-5,1 \pm 7,4+$ & $1,16 \pm 2,45 *$ & $0,75 \pm 1,55$ \\
\hline Flexibilidade Lombar (cm) & $22,7 \pm 9,4$ & $24,0 \pm 9,5$ & $1,15 \pm 4,60 *$ & $1,62 \pm 5,77$ \\
\hline
\end{tabular}

IMC = índice de massa corporal; ICQ = índice cintura-quadril; $P A=$ pressão arterial; $F C=$ frequência cardíaca; Cardio $=$ cardiorrespiratória; $M M S S=$ membros superiores; $\mathrm{ABD}=$ abdominal; $\mathrm{MMII}=$ membros inferiores. + = Diferente do grupo $S(P<0,05) ;{ }^{*}=$ Diferença entre a avaliação e a reavaliação $(P<0,05)$.

de membros inferiores, mas tinham menor flexibilidade lombar. $\mathrm{Na}$ reavaliação (Tabela 3), a PAS e a PAD diminuíram e a FC aumentou apenas nos homens. Por outro lado, a aptidão cardiovascular, a potência abdominal e a flexibilidade de ombros e lombar aumentaram significantemente apenas nas mulheres, enquanto que a flexibilidade de ombros tendeu a aumentar nos homens.

Em relação à idade, na avaliação, o grupo ID apresentou PAS significantemente maior que o grupo MI, mas a FC, aptidão cardiovascular, força de membros inferiores e flexibilidade de ombros foram significantemente menores neste grupo. $\mathrm{Na}$ reavaliação (Tabela 3), o grupo MI apresentou redução do peso e do IMC, e aumento da aptidão cardiorrespiratória, potência abdominal e flexibilidade de ombro e lombar. Já o grupo ID apresentou redução da PAD e aumento da aptidão cardiorrespiratória e da flexibilidade de ombro.

Considerando-se o nível de AF, na avaliação, os sujeitos dos grupos NA e A foram semelhantes, exceto pela colesterolemia, que foi significantemente maior no grupo NA. Na reavaliação (Tabela 3), no grupo NA, houve diminuição da massa corporal e do IMC, e aumento da aptidão aeróbica, potência abdominal, força de membros inferiores e flexibilidade de ombros. Já no grupo A, houve redução da PAD e aumento da aptidão cardiorrespiratória e da flexibilidade de ombros, além de uma tendência a aumentar a flexibilidade lombar. 
Tabela 3 - Diferenças nas variáveis antropométricas, hemodinâmicas, metabólicas e de aptidão física medidas na avaliação e reavaliação no grupo que seguiu as orientações, subdividido pelo sexo, idade (meia idade-MI e idosos-ID) e nível de atividade física (ativos-A e não ativos-NA).

\begin{tabular}{lcccccc}
\hline & Mulher & Homem & MI & ID & A & NA \\
\hline Massa & $-0,7 \pm 2,9$ & $-0,9 \pm 2,4$ & $-1,4 \pm 3,7^{*}$ & $-0,3 \pm 1,7$ & $-0,4 \pm 2,8$ & $-1,2 \pm 2,6^{*}$ \\
\hline IMC & $-0,2 \pm 1,2$ & $-0,3 \pm 0,7$ & $-0,5 \pm 1,4^{*}$ & $-0,1 \pm 0,7$ & $-0,1 \pm 1,0$ & $-0,5 \pm 1,0^{*}$ \\
\hline Cintura & $-0,5 \pm 5,9$ & $-1,0 \pm 3,9$ & $-0,5 \pm 5,4$ & $-0,8 \pm 5,3$ & $-0,3 \pm 4,6$ & $-1,2 \pm 6,2$ \\
\hline CQ & $-0,6 \pm 3,2$ & $-0,8 \pm 3,4$ & $-0,8 \pm 3,4$ & $-0,6 \pm 3,1$ & $-0,4 \pm 3,3$ & $-1,0 \pm 3,2$ \\
\hline ICQ & $0,01 \pm 0,07$ & $-0,01 \pm 0,05$ & $0,01 \pm 0,05$ & $0,00 \pm 0,07$ & $0,00 \pm 0,06$ & $0,00 \pm 0,07$ \\
\hline GLI & $-0,6 \pm 12,5$ & $2,2 \pm 11,2$ & $-0,7 \pm 12,9$ & $0,9 \pm 11,6$ & $0,5 \pm 10,5$ & $0,1 \pm 14,3$ \\
\hline COL & $-3,1 \pm 34,9$ & $-0,6 \pm 31,0$ & $-9,7 \pm 30,6$ & $2,3 \pm 34,5$ & $5,3 \pm 29,7$ & $-12,3 \pm 35,8$ \\
\hline PAS & $-0,3 \pm 13,1$ & $-7,4 \pm 12,7^{*}$ & $-1,7 \pm 12,6$ & $-3,3 \pm 13,9$ & $-1,8 \pm 14,3$ & $-3,9 \pm 11,9$ \\
\hline PAD & $-0,9 \pm 7,3$ & $-5,3 \pm 8,9^{*}$ & $-1,8 \pm 9,8$ & $-2,7 \pm 6,9^{*}$ & $-2,6 \pm 7,6^{*}$ & $-2,1 \pm 8,8$ \\
\hline FC & $-0,5 \pm 8,0$ & $3,1 \pm 8,1^{*}$ & $-0,2 \pm 10,6$ & $1,3 \pm 6,1$ & $0,7 \pm 8,2$ & $0,6 \pm 8,2$ \\
\hline Cardio & $12,3 \pm 18,1^{*}$ & $6,1 \pm 16,6$ & $10,6 \pm 18,3^{*}$ & $10,1 \pm 17,6^{*}$ & $9,2 \pm 16,6^{*}$ & $12,1 \pm 19,7^{*}$ \\
\hline Pot. MMSS & $0,3 \pm 4,5$ & $-0,2 \pm 3,8$ & $0,8 \pm 4,5$ & $-0,4 \pm 4,1$ & $-0,4 \pm 4,1$ & $0,9 \pm 4,5$ \\
\hline Pot. ABD & $2,0 \pm 4,0^{*}$ & $0,0 \pm 6,0$ & $2,9 \pm 3,3^{*}$ & $0,2 \pm 5,4$ & $-0,1 \pm 4,6$ & $3,6 \pm 4,4^{*}$ \\
\hline Força MMII & $0,3 \pm 3,2$ & $1,1 \pm 3,9$ & $0,7 \pm 4,0$ & $0,4 \pm 2,9$ & $-0,1 \pm 3,4$ & $1,6 \pm 3,1^{*}$ \\
\hline F. Ombros & $1,10 \pm 2,05^{*}$ & $1,32 \pm 3,26$ & $1,53 \pm 1,98^{*}$ & $0,91 \pm 2,72^{*}$ & $1,05 \pm 2,46^{*}$ & $1,33 \pm 2,47 *$ \\
\hline F. Lombar & $0,98 \pm 4,24^{*}$ & $1,50 \pm 5,37$ & $1,53 \pm 4,64^{*}$ & $0,89 \pm 4,61$ & $1,20 \pm 4,75$ & $1,07 \pm 4,46$ \\
\hline
\end{tabular}

* = Diferença entre a avaliação e a reavaliação $(P<0,05)$. Massa corporal $(\mathrm{kg})$, índice de massa corporal $(\mathrm{IMC}, \mathrm{kg} / \mathrm{m} 2)$, circunferência de cintura $(\mathrm{CC}, \mathrm{cm})$, circunferência do quadril $(\mathrm{CQ}, \mathrm{cm})$; índice cintura-quadril (ICQ), glicemia $(\mathrm{GLI}, \mathrm{mg} / \mathrm{dl})$, colesterolemia $(\mathrm{COL}, \mathrm{mg} / \mathrm{dl})$, pressão arterial sistólica (PAS, $\mathrm{mmHg}$ ), pressão arterial diastólica (PAD, $\mathrm{mmHg}$ ), frequência cardíaca ( $\mathrm{FC}, \mathrm{bpm})$, aptidão cardiorrespiratória (Cardio, passadas), potência de membros superiores (Pot.MMSS, repetições), potência abdominal (Pot.ABD, repetições), força de membros inferiores (Força MMII, cm), flexibilidade ombros (F.ombros, $\mathrm{cm}$ ) e flexibilidade lombar (F.lombar, $\mathrm{cm}$ ).

\section{DISCUSSÃO}

Os principais achados deste estudo foram que a prescrição de caminhada sem a supervisão direta da execução foi efetiva em promover modificações benéficas em vários parâmetros da saúde cardiovascular e da aptidão física dos frequentadores de um parque público. Os efeitos sobre a saúde foram, preferencialmente, verificados nas variáveis antropométricas e, em algumas situações, em parâmetros cardiovasculares. Quanto à aptidão física, os principais efeitos foram o aumento da aptidão cardiorrespiratória e da flexibilidade. Além disso, de forma geral, os maiores efeitos foram obtidos em indivíduos que inicialmente eram NA, nas mulheres e nos sujeitos mais jovens.

A efetividade do programa foi comprovada pelo fato das pessoas que seguiram as orientações dadas, mesmo que parcialmente, terem obtido efeitos positivos nos diversos parâmetros avaliados enquanto que nenhuma alteração benéfica foi verificada nos indivíduos que não seguiram a prescrição.

Em relação aos parâmetros antropométricos, houve diminuição da massa corporal e do IMC apenas no grupo S, principalmente, nos indivíduos dos subgrupos NA e de MI. Este resultado está de acordo com o reportado pela literatura sobre o efeito do treinamento não supervisionado na composição corporal ${ }^{16}$. Este efeito pode se dever ao aumento do gasto calórico pela AF, que pode auxiliar no controle da obesidade ${ }^{23}$. Este aumento deve ter sido mais evidente nos indivíduos inicialmente menos ativos, explicando o maior efeito neste subgrupo. Entretanto, a redução da massa corporal e do IMC também pode se dever a mudanças nos hábitos alimentares, que não foram controladas neste estudo. 
Em relação aos índices metabólicos, embora não tenha havido redução da glicemia no grupo S, houve aumento no grupo NS, de modo que a prática de atividade física e/ou a prescrição adequada pode ter impedido um aumento da resistência à insulina, ajudando no controle glicêmico ${ }^{5}$. Porém, a falta de controle da dieta pode ter impedido um maior efeito no grupo $\mathrm{S}$.

Diferentemente do esperado ${ }^{15,16}$, poucas alterações foram evidenciadas nas variáveis cardiovasculares com o treinamento não supervisionado. A PAD diminuiu nos homens, nos ativos e nos idosos, enquanto que a PAS diminuiu apenas nos homens. O pequeno efeito hipotensor pode ser explicado pelo baixo nível inicial da PA, pois é sabido que o efeito hipotensor do EF relaciona-se diretamente com o nível inicial da $\mathrm{PA}^{24}$. Assim, como vários indivíduos eram normotensos e muitos dos hipertensos faziam uso de medicamentos anti-hipertensivos, os valores iniciais da PA não eram elevados, o que pode explicar o reduzido efeito hipotensor do EF. Colaborando com esta hipótese está o fato de que nos homens, que apresentavam maior PA, a queda tanto da PAS quanto da PAD foi evidenciada.

Quanto à aptidão física, a aptidão cardiorrespiratória aumentou no grupo $\mathrm{S}$ como um todo, o que também foi evidenciado nos subgrupos analisados (exceto nos homens). Estas respostas podem ser explicadas pelo tipo de prescrição adotada, pois a caminhada de moderada intensidade e longa duração caracteriza um exercício aeróbico, que trás benefícios cardiorrespiratórios ${ }^{10}$.

A flexibilidade, principalmente de ombros, mas também lombar, melhorou em quase todos os grupos que seguiram as recomendações, o que deve ter ocorrido em função dos sujeitos receberem orientações complementares para realizar exercícios de alongamento ou por participarem das aulas de alongamento oferecidas.

A força de membros inferiores aumentou apenas no subgrupo NA. Apesar do exercício aeróbico não ser considerado um estímulo adequado para a melhora da força ${ }^{25}$, é possível que o pequeno estímulo dado pela caminhada no subgrupo NA já tenha sido suficiente para esta melhora, como já foi observado em idosos sedentários ${ }^{26}$. Por outro lado, como esperado, a potência de membros superiores não se modificou em nenhum dos grupos e subgrupos, pois a caminhada não estimula o aumento desta potência. Desta forma, a ausência de efeito sobre este parâmetro reforça que os resultados obtidos nas outras variáveis de aptidão física se deveram efetivamente à caminhada e não à adaptação à testagem, passagem do tempo ou outros aspectos, que aumentariam também a potência de membros superiores.

É interessante observar que a taxa de retorno para a reavaliação neste estudo foi de 30\%. Por se tratar de um projeto populacional com participação voluntária, era esperado um retorno menor, visto que a adesão a programas supervisionados é de $50 \%$ em 6 a 12 meses $^{27}$. Cabe ressaltar, que dos indivíduos que fizeram a reavaliação, $79 \%$ disseram ter seguido, pelo menos em parte, as orientações, sendo, a falta de tempo o principal motivo alegado pelos que não seguiram. De fato, este costuma ser o principal motivo para a não adesão a programas de $\mathrm{AF}$ em diferentes contextos ${ }^{28}$.

Este estudo apresenta diversas limitações que se relacionam diretamente com seu intuito de se avaliar uma situação real de atuação. Desta forma, foi impossível fazer um grupo controle adequado, pois as pessoas procuravam o projeto para se exercitar e, portanto, sugerir que elas não o fizessem seria antiético. A ausência deste grupo obrigou que o controle das variáveis intervenientes fosse feito por um grupo que relatou não ter seguido as recomendações. Porém, este grupo representa, na realidade, apenas uma parcela das pessoas que não seguiram as instruções, 
pois muitos simplesmente não retornaram para a reavaliação. Assim, os grupos $\mathrm{S}$ e NS foram avaliados separadamente, pois representam grupos com perdas de seguimento distintas. Como o objetivo era avaliar uma situação real, a amostra foi formada por todos os indivíduos que se voluntariaram, o que resultou em diferenças na proporção de pessoas em diferentes faixas etárias. Além disso, por ser um projeto populacional, outros fatores intervenientes também não puderam ser controlados, como a alimentação, o nível inicial de condicionamento físico dos participantes e o tempo entre avaliação e a reavaliação. Da mesma forma, os testes utilizados precisavam ser simples e aplicáveis num espaço pequeno, de modo que foram escolhidos testes com estas características apesar de outros testes poderem trazer informações adicionais. Para finalizar, a qualidade da aplicação dos testes não foi checada neste estudo, mas o treinamento dos monitores e a ausência de diferenças no grupo que não seguiu a prescrição sugerem que os testes foram bem conduzidos.

Apesar destas limitações, a grande contribuição deste estudo foi demonstrar que mesmo em condições reais de intervenção populacional, a prática regular de caminhada sem a supervisão direta da execução trouxe benefícios, sendo importante destacar que estes benefícios foram conseguidos mesmo em indivíduos já ativos inicialmente, refletindo a efetividade do programa. Desta forma, este tipo de programa (prescrição de caminhada sem supervisão da prática), que permite atender um grande número de pessoas, pode ser utilizado como uma estratégia de saúde pública para promover a prática segura de EF em locais públicos.

\section{CONCLUSÃO}

Um programa de orientação/prescrição de caminhada sem supervisão direta da execução pode ser efetivo para melhorar parâmetros de saúde cardiovascular e de aptidão física, desde que as orientações sejam seguidas, pelo menos em parte. Estes efeitos foram, principalmente, evidenciados em variáveis antropométricas, na aptidão cardiorrespiratória e na flexibilidade. Além disso, pessoas não ativas, de meia idade e mulheres apresentaram efeitos mais expressivos. Embora menos expressivos indivíduos ativos também tiveram benefícios. Estes dados reforçam a importância da presença de profissionais de AF em locais públicos para orientar a prática de EF.

\section{Agradecimentos}

Os autores agradecem o apoio da administração do Parque Fernando Costa e da Associação de Amigos e Ambientalistas do Parque da Água Branca (ASSAMA$\mathrm{PAB}$ ) para o desenvolvimento deste projeto. Este estudo recebeu apoio financeiro da Pró-Reitoria de Cultura e Extensão da USP.

\section{Contribuição dos autores}

Do primeiro ao sexto autor, todos participaram da coleta dos dados e tabulação, o primeiro e o segundo autor foram os principais responsáveis pela escrita e análise estatística dos dados. Estas foram revisadas por todos os autores que em algum momento contribuíram intelectualmente no desenvolvimento do estudo. Além disso, as três últimas autoras, a Prof ${ }^{\mathrm{a}}$ Teresa Bartholomeu, a Prof ${ }^{\mathrm{a}} \mathrm{Dr}^{\mathrm{a}}$ Tais Tinucci e a Prof ${ }^{a}$ Dra $^{a}$ Cláudia Lúcia de Moraes Forjaz foram responsáveis pela ideia do estudo e orientação desde a coleta até a escrita do artigo. 


\section{REFERÊNCIAS BIBLIOGRÁFICAS}

1. Ministério da Saúde. Painel de Indicadoteres do SUS. Brasília: Ministério da Saúde, 2006.

2. Vigitel Brasil 2011. Vigilância para Fatores de Risco e Proteção contra Doenças Crônicas por Inquérito Telefônico. Brasília: Ministério da Saúde, 2012.

3. Autenrieth CS, Baumert J, Baumeister SE, et al. Association between domains of physical activity and all-cause, cardiovascular and cancer mortality. Eur J Epidemiol 2011;26:91-9.

4. Cornelissen VA, Fagard RH. Effects of endurance training on blood pressure, blood pressureregulating mechanisms, and cardiovascular risk factors. Hypertension 2005;46:667-75.

5. Sigal RJ, Kenny GP, Wasserman DH, Castaneda-Sceppa C, White RD. Physical activity/ exercise and type 2 diabetes: a consensus statement from the American Diabetes Association. Diabetes Care 2006;29:1433-8.

6. Leon AS, Sanchez OA. Response of blood lipids to exercise training alone or combined with dietary intervention. Medicine and science in sports and exercise 2001;33:S502-15; discussion S28-9.

7. Heath GW, Parra DC, Sarmiento OL, et al. Evidence-based intervention in physical activity: lessons from around the world. Lancet 2012;380:272-81.

8. Matsudo V, Matsudo S, Andrade D, et al. Promotion of physical activity in a developing country: the Agita São Paulo experience. Public Health Nutr 2002;5:253-61.

9. Forjaz CL, Tinucci T, Bartholomeu T, et al. Assessment of the cardiovascular risk and physical activity of individuals exercising at a public park in the city of sao paulo. Arq Bras Cardiol 2002;79:35-50.

10. American College of Sports Medicine. ACSM's guidelines for exercise testing and prescription. Philadelphia: Lippincott Williams \&Wilkins, 2010.

11. Balady GJ, Chaitman B, Driscoll D, et al. Recommendations for cardiovascular screening, staffing, and emergency policies at health/fitness facilities. Circulation 1998;97:2283-93.

12. Roque T PP, Martins V, Bartholomeu T, Forjaz C. Triagem do risco cardiovascular para a prática de exercícios físcos nos frequentadores de um parque público. Coleção Pesquisa em Educação Física 2007;6:507-14.

13. Araujo L, Bartholomeu T, Tinucci T, Forjaz CLM. Perfil da Prática de atividade física dos frequentadores de um parque público de São Paulo. Rev Socesp Sup 2011;21:5-14 S04.

14. Forjaz CLM, Modesto BT, Bartholomeu T, Costa LAR, Tinucci T. PROJETO EXERCÍCIO E CORAÇÃO: UMA DÉCADA A SERVIÇO DA COMUNIDADE. Revista Cultura e Extensão USP 2011;6:61-9.

15. Carlson JJ, Johnson JA, Franklin BA, VanderLaan RL. Program participation, exercise adherence, cardiovascular outcomes, and program cost of traditional versus modified cardiac rehabilitation. Am J Cardiol 2000;86:17-23.

16. Nunes AP, Rios AC, Cunha GA, Barretto AC, Negrao CE. The effects of nonsupervised exercise program, via internet, on blood pressure and body composition in normotensive and prehypertensive individuals. Arq Bras Cardiol 2006;86:289-96.

17. Morris JN, Hardman AE. Walking to health. Sports Med. [Review] 1997;23:306-32.

18. Rikli R, Jones C. Development and validation of a functional fitness test for communityresiding older adults. J Aging and Phys Act 1999;7:129-61.

19. Soares J, Sessa M. Medidas de força muscular. In: Matsudo V, editor. Testes em ciências do esporte. São Caetano do Sul: CELAFISCS, 1995:73-7.

20. American Heart Association. Standards for adult exercise testing laboratories. American Heart Association Subcommittee on Rehabilitation, Target Activity Group. Circulation 1979;59:421A-30A passim.

21. Canadian CSEP. The Canadian Physical Activity, Fitness \& Lifestyle Approach: CSEP Health-Related Appraisal \& Counseling Strategy. Ottawa - ON: Health Canada, 2003.

22. Pollock ML, Gaesser GA, Butcher JD, et al. American College of Sports Medicine Position Stand. The recommended quantity and quality of exercise for developing and maintaining cardiorespiratory and muscular fitness, and flexibility in healthy adults. Medicine and science in sports and exercise. [Review] 1998;30:975-91.

23. Faselis C, Doumas M, Panagiotakos D, et al. Body mass index, exercise capacity, and mortality risk in male veterans with hypertension. American journal of hypertension 2012;25:444-50. 
24. Pescatello LS, Franklin BA, Fagard R, et al. American College of Sports Medicine position stand. Exercise and hypertension. Medicine and science in sports and exercise. [Review] 2004;36:533-53.

25. de Souza EO, Tricoli V, Franchini E, et al. Acute effect of two aerobic exercise modes on maximum strength and strength endurance. Journal of strength and conditioning research / National Strength \& Conditioning Association. [Comparative Study Randomized Controlled Trial] 2007;21:1286-90.

26. Ferketich AK, Kirby TE, Alway SE. Cardiovascular and muscular adaptations to combined endurance and strength training in elderly women. Acta physiologica Scandinavica. [Research Support, U.S. Gov't, P.H.S.] 1998;164:259-67.

27. Dishman RK. Advances in Exercises adherence. Champaingn: Human Kinectis, 1994.

28. Silva SG, Silva MC, Nahas MV, Viana SL. [Variables associated with leisure-time physical inactivity and main barriers to exercise among industrial workers in Southern Brazil]. Cadernos de saude publica / Ministerio da Saude, Fundacao Oswaldo Cruz, Escola Nacional de Saude Publica 2011;27:249-59.

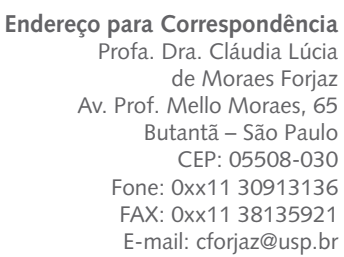

Recebido 23/10/2012

Revisado 29/10/2012 $11 / 01 / 2013$

Aprovado 13/01/2013 